\title{
U⿱宀⿻三丨口
}

\section{CAPTURE - Extending the Scope of Self-Localization in Indoor Positioning Systems}

Cullen, G., Curran, K., Santos, J. A., Maguire, G., \& Bourne, D. (2015). CAPTURE - Extending the Scope of Self-Localization in Indoor Positioning Systems. In Unknown Host Publication IEEE. https://doi.org/10.1109/IPIN.2015.7346777

Link to publication record in Ulster University Research Portal

\section{Published in:}

Unknown Host Publication

Publication Status:

Published (in print/issue): 13/10/2015

DOI:

10.1109/IPIN.2015.7346777

\section{Document Version}

Author Accepted version

\section{General rights}

Copyright for the publications made accessible via Ulster University's Research Portal is retained by the author(s) and / or other copyright owners and it is a condition of accessing these publications that users recognise and abide by the legal requirements associated with these rights.

\section{Take down policy}

The Research Portal is Ulster University's institutional repository that provides access to Ulster's research outputs. Every effort has been made to ensure that content in the Research Portal does not infringe any person's rights, or applicable UK laws. If you discover content in the Research Portal that you believe breaches copyright or violates any law, please contact pure-support@ulster.ac.uk. 


\section{CAPTURE - Extending the Scope of Self- Localization in Indoor Positioning Systems}

\author{
Gary Cullen, Kevin Curran, Jose Santos \\ Intelligent Systems Research Centre, \\ University of Ulster, Magee College, \\ Derry, United Kingdom \\ Gary.Cullen@1yit.ie
}

\author{
Gearoid Maguire, Denis Bourne \\ Letterkenny Institute of Technology, \\ Co. Donegal, Ireland
}

\begin{abstract}
The concept of devices cooperatively assisting with the localization of other devices in either the indoor and outdoor arena is not a new phenomenon. The primary focus of research into such a theory is however limited to solving the problem of localization accuracy. This paper outlines our Cooperatively Applied Positioning Techniques Utilizing Range Extension (CAPTURE) system which aims to provide a solution to the current range limitations of an Indoor Position System (IPS). These range limitations are the culmination of well documented difficulties of localizing using wireless signals Non-Line of Sight (NLOS) environments. The coverage of a localization solution is still a challenging issue in the indoor environment. In this paper we implement a version of CAPTURE that uses Wi-Fi Direct and Bluetooth Low Energy (Bluetooth LE 4.0) that takes advantage of mobile devices at the outer limits of an IPS to help extend its reach into blind spots, where devices cannot be located. CAPTURE is evaluated using a live test environment, where range estimations are captured between cooperating devices. These range estimations are filtered before being placed into a trilateration algorithm to position lost devices. Finally the accuracy of CAPTURE is presented, demonstrating the achievable benefits of implementing CAPTURE as a solution to the problem of coverage in an Indoor environment.
\end{abstract}

Keywords-Localization; Indoor Positioning Systems; Indoor Localization; Geographical Positioning; wireless; Wi-Fi; Bluetooth; Cooperative Localization; Collaborative Positioning; Self-Positioning

\section{INTRODUCTION}

The indoor location problem has been around for many years and has motivated a great deal of research into finding a solution. Cooperative solutions have made up a significant contribution of this research. Cooperation among devices to self-locate requires a key prerequisite - there must be an adequate number of devices willing to assist in locating a lost device. The proliferation of tablet devices and Smartphones, fully loaded with Wi-Fi, Bluetooth and gyroscopes, somewhat address this need. The advent of the Internet of Things (IoT's) however, providing access to 100's of billions of devices [1] offers an even more fertile community of wirelessly connected smart objects in a connectivity ecosystem. The pace of innovation of wearable computing coupled with tumbling costs, mirrored in the consumer interest of the iWatch offers no sense of a drop off in access to these collaborative devices. Indeed the requirement for nomadic wearable devices such as the aforementioned to be locatable further exacerbates the need for an expansive solution to accurately locate in all areas of an indoor environment. Devices such as these were typically not designed with wireless network capability to merely, assist in locating other devices. Which although, is a great reuse of an existing technology, does however, throw up a secondary issue. If these devices are connected to the network, can we realistically conceive that they will disconnect from that network to connect in a Peer to Peer network to cooperatively assist in locating lost devices? Wi-Fi Direct offers the ability to be in both Ad-Hoc Mode and Infrastructure mode simultaneously [2], Bluetooth LE allows the Wi-Fi chip to remain connected to the network whilst transmitting. Therefore using CAPTURE to extend the range of an IPS using Wi-Fi Direct and Bluetooth LE capable devices allows the user to remain connected to their network whilst cooperatively assisting in locating other devices it can 'see'.

Generally, IPS implementations can be grouped as either exogenous or endogenous depending on the available infrastructure that can be employed to establish location information. An exogenous infrastructure implementation is typically oriented towards an IPS application. An endogenous solution however, is made up of infrastructure that has not been installed primarily for positioning reasons. Currently, one of the most popular techniques to locate devices in the indoor environment is to use the preinstalled Wireless Access Points (WAPs) which are used to provide wireless network access to mobile devices. Typically, good system implementations are those that achieve an appropriate balance between requirements, technological advances, and costs. Whilst utilizing an existing infrastructure, such as this offers many noble qualities, not least the reduced costs in procuring equipment to implement a solution, it does introduce some problems. The decision process behind the strategic positioning of such equipment to provide mobile network coverage does not fulfil the requirements of an Indoor Positioning System (IPS) to locate devices. Therefore it is inevitable that blind spots should exist in these ISP's. When deciding on the positioning of Wi-Fi equipment such as Wireless Access Points (WAPs) the typical focus of network designers was to provide the highest available throughput to the largest congregations of wireless network users at key areas within the building. The ability to locate devices within that environment was not necessarily to the fore in their decision process, leaving gaping holes in terms of coverage in some of the IPS's currently in- 
place. This coupled with some of the architectural barriers to the positioning of WAPs within a buildings infrastructure doesn't suggest a solution to this issue in the near future.

In the outdoor arena, apart from some natural obstacles such as overhead trees, cavernous areas and mountainous regions the satellites that make up the Global Positioning System (GPS) have a relatively clear, unobstructed view of the devices they need to locate. There are also some man made obstructions, such as the urban canyon phenomenon [3], that can obscure views in the outdoor world. These also provide barriers to accurate position estimation, but bear little resemblance to the many impediments that make up the indoor environment. The ability to locate in the outdoor world has more or less been solved as a problem through the many advances in GPS technology down through the years. The inability of a GPS signals to penetrate a building's infrastructure after propagating 22,000 Kilometers however, renders it more or less redundant as an indoor positioning solution. Considering we spend more time in the indoor arena, nearly $89 \%$ of the time according to a recent study [4] carried out in Canada, the need for a solution is evident.

Usually, distance estimations from more than one WAP to a lost device are required so that a positioning algorithm can provide a reasonable level of accuracy. Indeed, sometimes up to four are required to provide positioning estimations on a 3D plane. We aim to show in this paper how CAPTURE can be used in this scenario to augment an existing ISP, assisting in the positioning of devices that would traditionally be unlocatable. Using devices bundled with $\mathrm{Wi}-\mathrm{Fi}$ or Bluetooth antennae we will make up the required number of, or completely replace the WAPs required. CAPTURE utilizes Received Signal Strength Indicator (RSSI) readings from Wi$\mathrm{Fi}$, Bluetooth or Wi-Fi Direct enabled devices to estimate the range between mobile devices. The measurement of signal attenuation of these RSSI values between cooperating devices is used to gauge a propagation distance. These distance estimations are filtered, to remove any outliers, before being used as input to a trilateration positioning algorithm. Previous iterations of CAPTURE [5, 6] have used both Wi-Fi and Bluetooth 2.0 to assist in the cooperative position of devices that are beyond the range of standard IPS's. In this paper we propose to use both Bluetooth 4.0 (Bluetooth LE (Low Energy)) and Wi-Fi Direct to find the location of remote devices.

Convincing other devices to cooperatively assist in locating lost devices would be impossible if, as part of that cooperation the assisting devices had to sacrifice copious amounts of battery power. In this paper, we will also provide an evaluation of the typically battery consumption when a device is utilized in a CAPTURE implementation. This will provide further evidence of the use of CAPTURE as a solution to the ranging problem.

Consider the following scenario, 'Bob' is sitting in the far end of the airport lounge reading his newspaper on his tablet and is considering ordering food. He has network connectivity and can see online that his flight is due to leave on time. Bob has been to this airport before but is unfamiliar with the time it would take to get to his specific departure gate, or which area he has to navigate his way through security. The airports IPS could assist with this, but he only has visibility of one WAP. This provides a robust network connection but is incapable of positioning Bob within the airport. Sue is in the airport café some 45 meters to the west of Bob, Sue's phone can be 'seen' by 3 different WAPs within the airports network and can be located to within 2 meters of her current position, via the inhouse IPS. Sue's phone can also 'see' Bob's tablet. The drinks vending machine in the main hall is 25 meters to the north of Bob, because of its location in the main hall it has access to 7 WAPs that are utilized in the airports IPS. This smart device also has a wireless Network Interface Card (NIC) allowing it to connect to the airport inventory system providing minute by minute updates on its current stock levels. But more importantly it is positioned within the network IPS. The 25 meter distance to Bob's tablet is a simple hop, well within its read range. In a normal scenario Bob would be beyond the range of the airports IPS, but because CAPTURE can utilize the known positions of Sue's phone, the drinks vending machine and the WAP that Bob can currently 'see', Bob can be positioned. CAPTURE takes these devices that know their position and estimates range distances from Bobs lost device to them. These range estimates are then placed into a trilateration algorithm to position Bob within the airport. CAPTURE provides a position estimate relative to the devices locating it, which can then be mapped onto a global overview of the airport IPS. Bob can now see that he is 15 minutes from the departure gate, he is advised to go via the security area just behind the lounge. Bob orders the duck, all is good.

\section{CAPTURE - SYSTEM MODEL}

Here we describe the overall system model used to implement the CAPTURE solution. CAPTURE provides the ability to locate a lost device relative to devices that were used to assist in its localization. If these devices were originally positioned with an IPS this position can be translated to Cartesian coordinate values on a 2D plane. There is no need for a calibration stage with CAPTURE as would be required with the classical fingerprinting model. CAPTURE uses only real time RSSI values, providing a robust opportunistic solution in dynamic environments. Literature within the realm of Location Based Systems frequently use terms such as Anchor or Anchor Nodes to describe devices that help to determine the position of lost or unknown devices. The term anchor elicits a perception of a static or permanent device, which in a cooperative solution these devices most certainly are not. For this reason we will use the term reference device when describing devices that assist in the positioning of lost or unknown devices.

Fundamental to any position estimation algorithm is the ranging technique that is employed to gauge the distance from the transmitting device(s) to the receiving device(s). This is determined using a given metric, for example the length of time it takes a signal to propagate the distance from the transmitter to the receiver. Issues relating to the small scale fading of signals due to reflection, refraction, absorption, diffraction and scattering on obstacles in the indoor environment are non-trivial and have been well documented in 
the following literature [7-9]. A high percentage of location algorithms use range measurements or angle measurements to estimate distance between a lost device and a particular reference device. CAPTURE uses the Received Signal Strength Indicator (RSSI) to estimate range between devices. This range estimation is then used as input to a trilateration algorithm to ascertain the Cartesian coordinate values of the lost device. In order for CAPTURE to be able to cooperatively locate a lost device within a network, there must be at least 3 reference devices within sight of the lost device. Each of these must have 'a prior' knowledge of their location within a preexisting localization solution. During the experiments, different configurations were modelled to mimic the different scenarios that could befall a lost device being positioned using CAPTURE. Initially 3 Smartphones were used to position the lost device, then two phones along with a WAP were used to provide range estimations as input. Finally RSSI readings from a Smart TV, equipped with a Wi-Fi card, a tablet and a Smartphone were used to represent the scenario described as Bob's scenario at the airport.

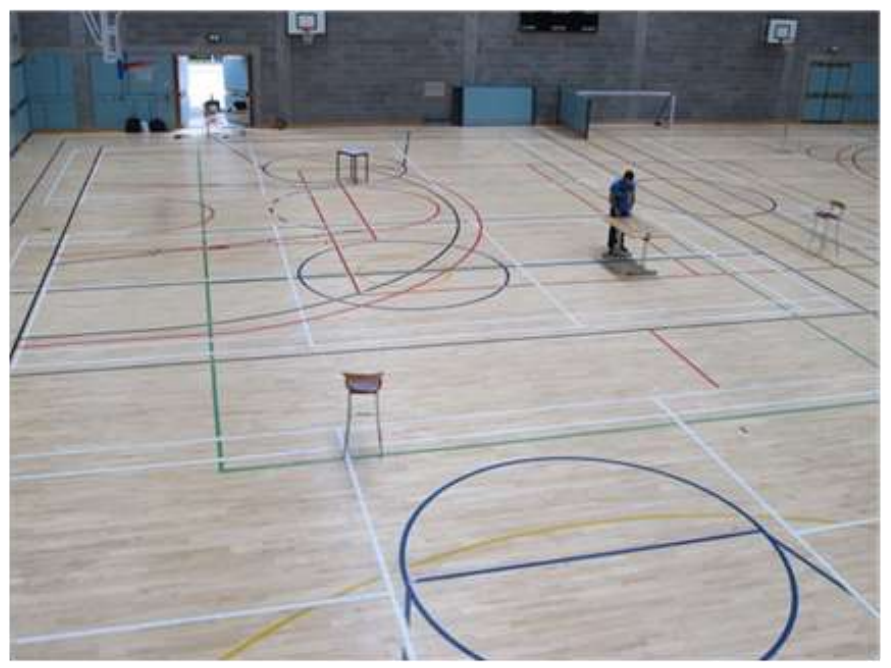

Figure 1: Test Environment

\section{EXPERIMENTAL TEST BED}

In this section, we will demonstrate the suitability of CAPTURE as a solution to the indoor ranging problem. We will back up this assertion with evidence based tests, carried out in a large campaign of measurements taken in a Sports Hall. The hall offers a $40 \mathrm{~m}$ wide diagonal testing environment, providing Line of Sight measurements for all tests, as can be seen in the image depicted in Figure 1. Each device used in the test is given a name (BSSID) TestDevice1, TestDevice2 for example. CAPTURE takes the RSSI readings of all available reference points, i.e. all devices it can 'see', but it filters out only the test devices selected by the user carrying out the tests. This is achieved via a lookup table mapping the MAC address of the device to the device name and allows us to work only with cooperating devices, allowing the use of only a specified device or a group of device during any given test. The experimental setup of CAPTURE was made up of 7 Samsung GT-S5310 Galaxy Pocket phones, running Google Android 2.2.1 on a $600 \mathrm{MHz}$ ARMv6, Adreno $200 \mathrm{GPU}$, Qualcomm
MSM7227 chipset. 3 of the phones were used as reference devices, the other phone acted as the lost device. All equipment used during the experiments were the same make and model ruling out any issues with diverse RSSI reads with different antenna types. Issues relating to varied reads with diverse antenna makes have been documented in the literature [10, 11]. Lisheng et al., [10] describe the bias being as much as 11.2 $\mathrm{dBm}$ out with different antenna types over a 25 meter read range. Although these issues describe scenarios relating to $\mathrm{Wi}$ Fi radio signals, it is the opinion of the author that these would have a negative impact on Bluetooth LE transmissions also. Other issues relating to the orientation of devices described in the following literature [12] were also considered during the testing phase.

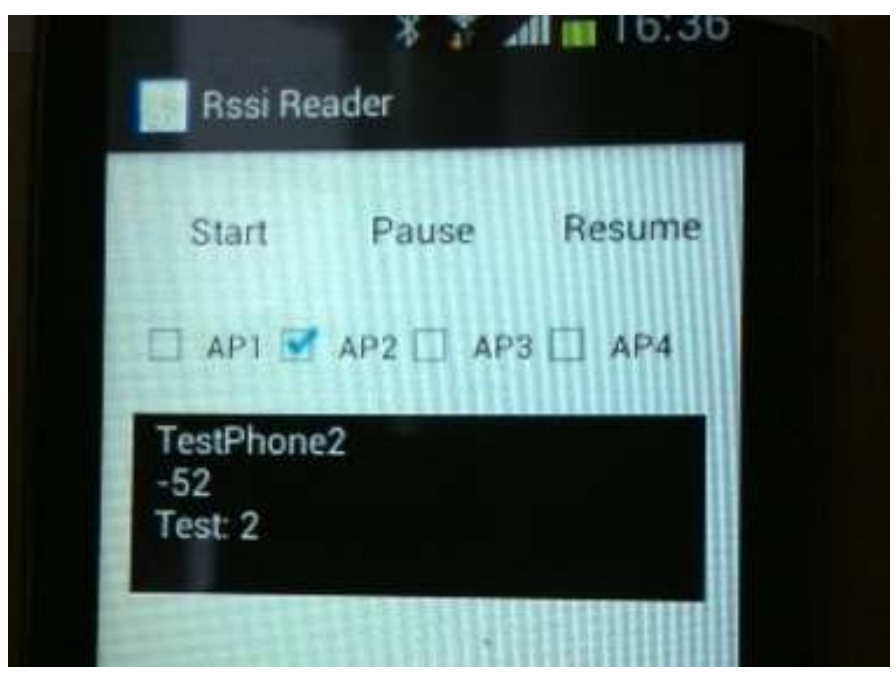

Figure 2: CAPTURE Client Interface

A MySQL Server version: 5.0.96 hosted on a Linux platform was used to store all data collected by the devices. The server was online and the mobile devices wrote directly to it as they recorded RSSI values from each other. The data was then passed through a low level filter to remove any outliers, before an average RSSI reading was calculated for each required ranging measurement, to be used in the trilateration algorithm to estimate the position of the lost device. A Dell Latitude E6440 iCore3 running Windows 7 Professional was used to develop the app to gather the RSSI from the phones. An algorithm was designed to convert this RSSI reading into a ranging measurement before a trilateration algorithm converted the ranging measurements into Cartesian coordinate values. We used the Eclipse IDE and Android Software Development Kit (SDK) for Android development and debugging, to develop the app.

\section{DATA COLLECTION AND PRESENTATION}

In this section we provide an overview of some of the data collected during the experiments that were carried out during this implementation of CAPTURE. An initial test was carried out to provide an average 1 meter read range for the ranging algorithm. This test involved over 500, 1 meter RSSI readings recorded at different locations throughout the test area. Any 
outliers were removed with a simple filter, this allowed for the accurate depiction of this reading to be used for the ranging algorithm. Additional tests were carried out to measure the accuracy of the RSSI readings and the computed range estimations achieved by the algorithm. The following table, Table, Table I. CAPTURE RSSI Readings, outlines some of the readings achieved during this initial test phase of the implementation.

TABLE I. CAPTURE RSSI READINGS

\begin{tabular}{|l|c|c|c|c|}
\hline \multicolumn{5}{|c|}{ RSSI Readings } \\
\hline Distance & $\mathbf{0}-\mathbf{5} \mathbf{~ m}$ & $\mathbf{0}-\mathbf{1 0} \mathbf{~ m}$ & $\mathbf{0}-\mathbf{1 5} \mathbf{~ m}$ & $\mathbf{0}-\mathbf{2 0} \mathbf{~ m}$ \\
\hline Average & -57.264 & -61.5652 & -69.5263 & -67.5662 \\
\hline Std. Dev & 0.4996 & 0.4 & 0.85346 & 0.48992 \\
\hline Estimate & 4.517 & 8.269 & 25.31 & 19.216 \\
\hline Distance & $\mathbf{0 - 2 5} \mathbf{~ m}$ & $\mathbf{0}-\mathbf{3 0} \mathbf{~ m}$ & $\mathbf{0}-\mathbf{3 5} \mathbf{~ m}$ & $\mathbf{0}-\mathbf{4 0} \mathbf{~ m}$ \\
\hline Average & -68.38 & -70.75 & -71.854 & -73.681 \\
\hline Std. Dev & 0.6884 & 0.9797 & 0.6803 & 0.7901 \\
\hline Estimate & 21.544 & 30.059 & 35.104 & 45.379 \\
\hline
\end{tabular}

\section{CONCLUSIONS}

A comprehensive solution to localization in the indoor arena poses a uniquely complex set of problems. Problems that have fostered growing interest, but for which, a single or indeed swift answer does not currently exist. The success of GPS has further exacerbated the need for an indoor representation. This paper introduced a novel architecture for cooperative localization, CAPTURE, a solution to extend the range of an Indoor Positioning System. The proposed approach provides an efficient reliable mechanism to plug into an in-situ solution. An implementation of CAPTURE was evaluated and tested which demonstrated the ability of CAPTURE to augment an existing indoor localization solution to locate mobile devices. Battery consumption was highlighted earlier in the paper, this will be adequately analyzed in a larger version of this paper. Three different scenarios were also outlined describing specific situations where typically devices could not be located using a standard solution. The first of these scenarios was, were only one WAP was reachable by the lost device, the second were the lost device could 'see' a WAP and a smart object of some description, and the final scenario were only mobile devices existed within range of the lost device. It is proposed that further experiments (in the coming months) will be implemented to replicate each scenario in detail. Each of these would be evaluated to establish the feasibility and accuracy of each which would further advocate CAPTURE as a solution to these problems.

\section{REFERENCES}

[1] G. Kortuem, F. Kawsar, D. Fitton, and V. Sundramoorthy, "Smart objects as building blocks for the Internet of things," Internet Computing, IEEE, vol. 14, pp. 44-51, 2010.

[2] W.-F. Alliance, "Wi-Fi Certified Wi-Fi Direct," White paper, October 2010 .

[3] M. Spangenberg, J. Y. Tourneret, V. Calmettes, and G. Duchateau, "Detection of variance changes and mean value jumps in measurement noise for multipath mitigation in urban navigation," in Signals, Systems and Computers, 2008 42nd Asilomar Conference on, 2008, pp. 11931197.

[4] C. G. Richardson, J. Memetovic, P. A. Ratner, and J. L. Johnson, "Examining gender differences in emerging tobacco use using the adolescents' need for smoking scale," Addiction, vol. 106, pp. 1846 1854, 2011.

[5] G. Cullen, K. Curran, J. Santos, G. Maguire, and D. Bourne, "CAPTURE - Cooperatively Applied Positioning Techniques Utilizing Range Extensions," in Indoor Positioning and Indoor Navigation (IPIN), 2014, 2014, pp. 248-254.

[6] G. Cullen, K. Curran, J. Santos, G. Maguire, and D. Bourne, "To wireless fidelity and beyond \& Beyond; CAPTURE, extending indoor positioning systems," in Ubiquitous Positioning Indoor Navigation and Location Based Service (UPINLBS), 2014, 2014, pp. 248-254.

[7] T. S. Rappaport, Wireless communications : principles and practice, 2nd ed. ed. Upper Saddle River, N.J. ; [Great Britain]: Prentice Hall PTR, 2002.

[8] S. S. Haykin and M. Moher, Modern wireless communications. Upper Saddle River, N.J.: Pearson/Prentice Hall, 2005.

[9] A. F. Molisch, Wireless communications, 2nd ed. ed. Oxford: John Wiley \& Sons, 2011.

[10] X. Lisheng, Y. Feifei, J. Yuqi, Z. Lei, F. Cong, and B. Nan, "Variation of Received Signal Strength in Wireless Sensor Network," in Advanced Computer Control (ICACC), 2011 3rd International Conference on, 2011, pp. 151-154.

[11] F. D. Rosa, X. Li, J. Nurmi, M. Pelosi, C. Laoudias, and A. Terrezza, "Hand-grip and body-loss impact on RSS measurements for localization of mass market devices," in Localization and GNSS (ICLGNSS), 2011 International Conference on, 2011, pp. 58-63.

[12] K. Kaemarungsi and P. Krishnamurthy, "Properties of indoor received signal strength for WLAN location fingerprinting," in Mobile and Ubiquitous Systems: Networking and Services, 2004. MOBIQUITOUS 2004. The First Annual International Conference on, 2004, pp. 14-23. 